\title{
Women in developing countries: assessing strategies for empowerment
}

R. Datta and J. Kornberg (editors); Lynne Rienner Publishers, Boulder, C0; 2002, Pbk ISBN $1588260399 £ 11.00$

There has been considerable discussion regarding theories behind, implementation of, and impacts of economic development programs. While development programs have improved conditions for citizens of developing nations, these programs do not equally benefit all, and often promote inequalities between the genders. Rekha Datta and Judith Kornberg, with their contributing authors, add to this discussion in Women in Developing Countries: Assessing Strategies for Empowerment which examines a variety of development programs on the empowerment of women. However, while the book is of tremendous value in terms of factual information, it falls short in contributing to systematic theory building.

Datta and Kornberg bring together authors who address a range of case and comparative studies relating to development policies and women. The cornucopia of studies presents the first challenge. There does not seem to be any logical rationale behind the choice of nations or cases included in the book, and the authors do not use a universal framework or theoretical approach. The lack of consistent criteria for case selection presents problems for comparability, as does the lack of any consistent theory or approach tying the chapters together. Methods used in this book range from narrative approaches, to case studies, to comparative studies of nations. While all methods help pinpoint factors that explain the success or failure of developmental programs, and the impact they have on empowering women, because each of the chapters functions as a stand-alone article rather than part of a bigger theoretical approach, the book, as a whole, falls short. In the end, there are eight chapters that raise more questions than they answer, and seem completely disconnected from each other and advancement of broader theory.

Datta and Kornberg also face difficulty in using empowerment as the basis of their evaluations. The first problem is their definition of empowerment. Datta and Kornberg assert empowerment is a process and an outcome, a contested concept, situational and relational, and can be differently defined, depending on the context. There are two problems with this approach to the definition of empowerment. First, defining empowerment as a process and an outcome allows us to define anything as empowering (or disempowering) as it is unclear at which point process ends and outcome begins, or what relationship an outcome may have to creating another process or outcome. Datta and Kornberg's definition leaves the door open to what constitutes empowerment.

The second problem with the definition of empowerment is the author's assertion that it is a situational term defined by culture, religion and context creating a 'mushy' dependent variable that undermines systematic theory building. By defining a key term too loosely, it becomes trapped in a sea of culture, and limits 
its scope and general applicability. In the end, defining a term in the context of culture in which it exists, prevents attributing the empowerment of women to anything more than culture, leaving unsatisfactorily answered the question of the impact development programs can and do have on women.

Datta and Kornberg's use of empowerment of women as a way to measure the success of development programs is an interesting approach. The success of development programs are usually measured in terms of change in income, education and literacy rates, and/or population growth rates - all measures that suggest a normative bias that development programs should equally affect all citizens, regardless of gender. However, as the studies in Datta and Kornberg's work suggest, this is often not the case. Development programs tend to benefit certain groups of individuals, and impact men and women in different ways, often to the detriment of women. Therefore, finding a way to isolate women in developing nations to determine how control over their own lives changes as a result of development programs is an interesting proposition. The question is how to evaluate development programs on an individual, rather than national, level. Datta and Kornberg have taken this challenge, and their book is a good first attempt. However, the broad definition these authors allow for their dependent variable presents many methodological problems which prevent it from being an appropriate way to engage in answering these kinds of questions.

Measuring the impact of development programs is at best difficult because multiple factors, such as history, culture, religion, geography, political and social systems, and norms, can intervene to impact a program's performance. Sorting out independent variables, and defining them clearly and consistently is a challenge to any scholar studying development. Causal relationships are hard to clearly identify, and determining how one factor influences another presents additional difficulties. Further, any work addressing development policies must ultimately engage in normative debate about whether the 'Western' way of development is the only path nations can pursue. These are all hard questions that have no easy answers, and there is no one way to research the difficult topic of economic development - new approaches are needed. While the notion of empowerment as a measure of developmental success is a valuable approach that should be pursued, it must be done in a more methodologically consistent manner if it is to help us better understand development in a worldwide context.

doi: $10.1057 /$ palgrave.fr. 9400192

Erin $\varepsilon$. Richards 